University of Nebraska - Lincoln

DigitalCommons@University of Nebraska - Lincoln

Publications from USDA-ARS / UNL Faculty

U.S. Department of Agriculture: Agricultural

Research Service, Lincoln, Nebraska

August 2002

\title{
Nitrogen and Dry Matter Distribution by Culm and Leaf Position at Two Stages of Vegetative Growth in Winter Wheat
}

Wally Wilhelm

University of Nebraska-Lincoln, wally.wilhelm@ars.usda.gov

G. S. McMaster

USDA-ARS, greg.mcmaster@ars.usda.gov

Dorothy M. Harrell

University of Nebraska-Lincoln, dharrell2@unl.edu

Follow this and additional works at: https://digitalcommons.unl.edu/usdaarsfacpub

Part of the Agricultural Science Commons

Wilhelm, Wally; McMaster, G. S.; and Harrell, Dorothy M., "Nitrogen and Dry Matter Distribution by Culm and Leaf Position at Two Stages of Vegetative Growth in Winter Wheat" (2002). Publications from USDAARS / UNL Faculty. 65.

https://digitalcommons.unl.edu/usdaarsfacpub/65

This Article is brought to you for free and open access by the U.S. Department of Agriculture: Agricultural Research Service, Lincoln, Nebraska at DigitalCommons@University of Nebraska - Lincoln. It has been accepted for inclusion in Publications from USDA-ARS / UNL Faculty by an authorized administrator of DigitalCommons@University of Nebraska - Lincoln. 


\title{
Nitrogen and Dry Matter Distribution by Culm and Leaf Position at Two Stages of Vegetative Growth in Winter Wheat
}

\author{
W. W. Wilhelm,* G. S. McMaster, and D. M. Harrell
}

\begin{abstract}
Knowledge of $\mathbf{N}$ and assimilate partitioning in wheat (Triticum aestivum $\mathrm{L}$.) improves management efficacy and crop model development. Our purpose was to describe $\mathbf{N}$ and dry matter distribution during vegetative growth of blades, sheaths, and internodes on four culms. Winter wheat grown at the Colorado State University Horticultural Farm was sampled at Haun Stage 5 and jointing. Samples were dried, weighed, and analyzed for $\mathrm{N}$. As the canopy developed and older tissue contributed more of total tissue, $\mathbf{N}$ concentration decreased although $\mathbf{N}$ mass and dry weight increased. Dry matter and $\mathrm{N}$ mass decreased from $\mathrm{MC}$ to $\mathrm{T} 1$ and $\mathrm{T} 2$ to $\mathrm{T11}$, while the reverse order was found for $\mathbf{N}$ concentration. Dry weight and $\mathbf{N}$ mass decreased as culm order increased (culm age decreased), because phytomers were smaller and fewer existed. Nitrogen concentration had the opposite trend because new tissue contained about $40 \mathrm{~g} \mathrm{~N} \mathrm{~kg}^{-1}$ but declined as the tissue aged to $30 \mathrm{~g} \mathrm{~N} \mathrm{~kg}^{-1}$. Initial growth of all tissues had concentrations $>50 \mathrm{~g} \mathrm{~N} \mathrm{~kg}^{-1}$ and at senescence declined to $19 \mathrm{~g} \mathrm{~N} \mathrm{~kg}^{-1}$. Phytomer positions on different culms tended to have similar $\mathbf{N}$ concentrations while identical phytomers on primary tillers tended to have greater dry weights than those on the MC and secondary tillers. Phytomers tended to increase in $\mathbf{N}$ concentration and mass and dry weight acropetally. Results show that viewing the canopy as the interplay of appearance, growth, interaction, and senescence of culms or phytomers can increase understanding of canopy $\mathbf{N}$ and dry weight dynamics.
\end{abstract}

$\mathrm{N}^{\mathrm{n}}$ ITROGEN has long been recognized as a critical nutrient for wheat (Miller, 1939). Work establishing adequate nutrient levels for crops has usually involved whole-canopy analysis (Baker and Tucker, 1973; Melsted et al., 1969), but researchers have increasingly recognized the importance and utility of analyzing individual plant components at various stages of growth to better understand how wheat develops and grows (Baker and Tucker, 1973; Karlen and Whitney, 1980; McMaster, 1997). Analysis of individual plant components, when done, has almost exclusively been based on pooling basic tissue types such as leaves, stems, and kernels from all shoots within the canopy. With the development of morphological naming schemes that uniquely identify

W.W. Wilhelm, USDA-ARS, Soil and Water Conservation Res. Unit, Univ. of Nebraska, Lincoln, NE 68583; G.S. McMaster, USDA-ARS, Great Plains Systems Res. Unit, P.O. Box E, Fort Collins, CO 80522; and D.M. Harrell, School of Natural Resource Sci., Univ. of Nebraska, Lincoln, NE 68583 (formerly, USDA-ARS, Soil and Water Conservation Res. Unit, Univ. of Nebraska, Lincoln, NE 68583). Joint contribution of USDA-ARS and Nebraska Agric. Res. Div. Published as Journal Series no.13482, Agric. Res. Div., Univ. of Nebraska. Received 25 Sept. 2001. *Corresponding author (wwilhelm1@unl.edu).

Published in Agron. J. 94:1078-1086 (2002). each plant component (Klepper et al., 1983; Wilhelm and McMaster, 1996), researchers can now communicate with greater detail and precision about phenomena occurring during wheat growth and development. The basic tissue types of leaves, stems, and kernels can now be partitioned as part of individually identified culms or as parts of phytomers. Phytomers are the fundamental building block of culms consisting of a node and tissues developed from it, including the blade, sheath, internode, and axillary bud (Wilhelm and McMaster, 1995). An advantage of this approach is that the canopy can be considered a dynamic population of culms or phytomers appearing, growing, interacting, and senescing over time, with the final result determining crop yield (Harper, 1977; McMaster, 1997).

In addition to the fact that little attention has been paid to $\mathrm{N}$ distribution within the wheat canopy, most work has emphasized distribution of $\mathrm{N}$ and assimilate during the reproductive phase and their effects on yield (Badaruddin et al., 1999; Demotes-Mainard et al., 1999). Unfortunately, far fewer reports exist on the content and distribution of $\mathrm{N}$ and dry matter in winter wheat during vegetative growth, the period during which most of the yield potential is established (Gregory et al., 1979, 1981; Waldren and Flowerday, 1979). Translocation of assimilates (Demotes-Mainard et al., 1999; Rawson and Hofstra, 1969; Wardlaw, 1967) and N (Bauer et al., 1987; Demotes-Mainard et al., 1999; Jeuffroy and Bouchard, 1999; McNeal et al., 1966) into wheat grain during grain filling is well documented. During grain filling, $\mathrm{N}$ content of nongrain tissue decreases while grain $\mathrm{N}$ content increases (Bauer et al., 1987; Boatwright and Haas, 1961; Campbell and Davidson, 1979; Karlen and Whitney, 1980; Spratt and Gasser, 1970). Although studies exist on the distribution of photosynthate in winter wheat (Thorne, 1982) and barley (Hordeum vulgare L.; Lauer and Simmons, 1985, 1988) canopies, more specific information on dry matter and $\mathrm{N}$ partitioning is needed to understand wheat canopy growth and development during the vegetative phase.

Descriptions of $\mathrm{N}$ distribution and dry weights among and within precisely identified culms and phytomers over the growing season would be useful in developing and testing simulation models of wheat development and

Abbreviations: GDD, growing degree-days; MC, main culm; SE, standard error of the mean; T1, primary tiller arising from the axillary bud of the first leaf on the MC; T2, primary tiller arising from the axillary bud of the second leaf on the MC; T11, secondary tiller arising from the axillary bud of first leaf on primary tiller T1. 
growth. As we develop increasingly detailed models of crop growth and development, incorporating better algorithms for translocation and use of $\mathrm{N}$ and assimilate within the plant is essential (Jones and Kiniry, 1986; McMaster et al., 1992; Rickman et al., 1996). Efficacy of fertilizer, irrigation, chemical application, and other crop management activities should also be enhanced with detailed knowledge of partitioning and translocation of $\mathrm{N}$ and assimilates over the life of the plant. Therefore, the purpose of this paper is to describe $\mathrm{N}$ and dry matter partitioning among uniquely identified wheat plant parts during vegetative growth.

\section{MATERIALS AND METHODS}

Hard red winter wheat (cv. Vona) was sown in a two-season study at the Colorado State University Horticultural Farm (41 $36^{\circ} \mathrm{N}$ lat, $104^{\circ} 59^{\prime} \mathrm{W}$ long) near Fort Collins, CO, on a Nunn clay loam soil (fine, smectitic, mesic Aridic Argiustolls). Soil tests at sowing indicated that all nutrients were well above recommended levels for wheat production in the region. Land used for this study was fallow for 14 mo immediately preceding sowing of each year (summer fallow). Sowing was on 17 Sept. 1986 and 23 Sept. 1987 with 30-cm row spacings. For this experiment, the field was divided into eight blocks, four used each season. Plots were arranged differently each season of the study and their sizes were $3 \mathrm{~m}$ (10 rows) wide by $30 \mathrm{~m}$ long in 1986, and $7.2 \mathrm{~m}$ (24 rows) wide by $15 \mathrm{~m}$ long in 1987. Since the primary focus of this paper is distribution of $\mathrm{N}$ concentration and content and dry matter among tissues and culms within the plant during vegetative development, effects of management treatments were accounted for in the statistical analysis. During the 1986-1987 season, two fertilizer treatments (0 and $134 \mathrm{~kg} \mathrm{~N} \mathrm{ha}^{-1}$ applied on 31 Mar. 1987) and two irrigation treatments (irrigation at late jointing [19 May 1987] and no irrigation) were applied in a factorial arrangement. The four treatments in 1987-1988 were single irrigation on 13 May 1988 (late jointing), single irrigation on 6 June 1988 (anthesis), irrigation on both 13 May and 6 June, and dryland (no irrigation). No $\mathrm{N}$ treatments were investigated during the 1987-1988 season. Each season, treatments were randomly assigned to one plot in each of four blocks in a randomized complete block design. Climatological data collected at a Class A weather station at the site are summarized in a previous publication (McMaster et al., 1994).

Emerging main culms and tillers were marked with loosely fitting loops of colored wire to facilitate culm and phytomer unit identification during sampling. The morphological naming scheme is that of Klepper et al. (1983). Plants were sampled 16 Dec 1986 (tillering), 18 May 1987 (late jointing), 11 Dec. 1987 (tillering), and 13 May 1988 (late jointing) for the analysis of vegetative growth. No management treatments had been applied before December of either season or May 1988; thus, on those sampling dates, all plots are considered replicates. In 1986-1987, only the fertilization treatment had been applied before the May sampling, resulting in eight replicates of two treatments (fertilizer application, control). In all analyses, classification variables of interest were culm identification (culm), phytomer unit (position), and tissue (blade, sheath, and internode).

At each sampling, we collected plants from a 0.5-m length of one row from each plot. Plants were gently washed free of soil and arranged according to number and size of tillers and height of culms; the five median plants were selected for analysis. All culms on the five plants were identified and a Haun scale for leaf number determined (Haun, 1973). Each culm was separated into individual blades, sheaths, and internodes (including the subtending node, but referred to as internodes in this paper). Tissues were dried at $65^{\circ} \mathrm{C}$ to constant weight and weighed. Irrigation treatment and culm effects on grain yield and yield components have been described previously (McMaster et al., 1994).

Only the main culms (MC), primary tillers $\mathrm{T} 1$ and $\mathrm{T} 2$, and the most frequently appearing secondary tiller, T11, were prepared for $\mathrm{N}$ analysis. Individual blades, sheaths, and internodes from those culms were analyzed for total $\mathrm{N}$ by the Dumas dry combustion technique using an automated Carlo Erba NA 1500 Series I N and C Analyzer ${ }^{1}$ (Carlo Erba Instruments, Milan, Italy) interfaced to a Europa tracer mass spectrometer (PDZ Europa Ltd., Cheshire, UK) as described by Schepers et al. (1989). If the mass of individual plant tissues was $>75 \mathrm{mg}$, the samples were ground to a fine powder (approximately 100 -mesh particle size) in a roller mill and approximately $5 \mathrm{mg}$ of the powder was placed into the capsule for analysis. If samples were between 2.5 and $8.5 \mathrm{mg}$, the entire organ (blade, sheath, or internode) was placed directly into the sample capsule. Organs $<2.5 \mathrm{mg}$ were combined with other samples of the same tissue, culm, and phytomer from one or more of the other four plants in the sample to obtain a combined weight of 2.5 to $8.5 \mathrm{mg}$ and analyzed. Samples between 8.5 and $75 \mathrm{mg}$ were combined with other samples in the same way and milled before analysis. Occasionally, identical tissues from adjacent phytomers had to be combined to obtain enough material for analysis. In these few instances, tissues from both phytomers were assigned the resulting $\mathrm{N}$ concentration. Samples that were not in an acceptable weight range and could not be combined with others to yield sufficient mass for analysis were not analyzed (1327 of 6404 samples; about $20 \%$ ). Nitrogen mass was calculated for each organ as the product of tissue mass and $\mathrm{N}$ concentration.

The SAS statistical package (SAS Inst., 1989) was used for data analysis. Values of tissue weight, $\mathrm{N}$ mass, and $\mathrm{N}$ concentration were averaged using the Means procedure for all plants in the subsample (up to five plants if $\mathrm{N}$ concentration was measured for all five). Separate analyses of variance (General Linear Models procedure) were performed for each tissue type and sampling date. Means for each culm and each position were calculated using the Means procedure because the irregularity in sample sizes caused the LSMeans procedure to produce nonestimable values. Standard errors (SE) were calculated for each mean; aggregate values were calculated using sums and means weighted by the fraction of plants bearing each tissue. Weighted sums were used to calculate $\mathrm{N}$ mass and dry weight totals for tissues, culms, phytomers, and whole plants; weighted means were used to calculate $\mathrm{N}$ concentrations and all means across seasons. Culm, phytomer, and tissue type effects were tested with the analysis of variance. When effects were significant ( $p<0.05$, unless stated otherwise), means were separated with the $t$-test.

\section{RESULTS AND DISCUSSION}

Fall and winter (September-March) of the 19861987 season were slightly cooler $\left(-0.2^{\circ} \mathrm{C}\right)$ and wetter $(+81 \mathrm{~mm})$ than average (30-yr mean); the spring and early summer (April-July) were slightly warmer $\left(0.2^{\circ} \mathrm{C}\right)$ and drier $(-27 \mathrm{~mm})$ than average. During the 1987-1988 season, the fall and winter (September-March) were

\footnotetext{
${ }^{1}$ Trade names mentioned are intended for the benefit of the reader and do not imply endorsement of any products by USDA-ARS or University of Nebraska.
} 

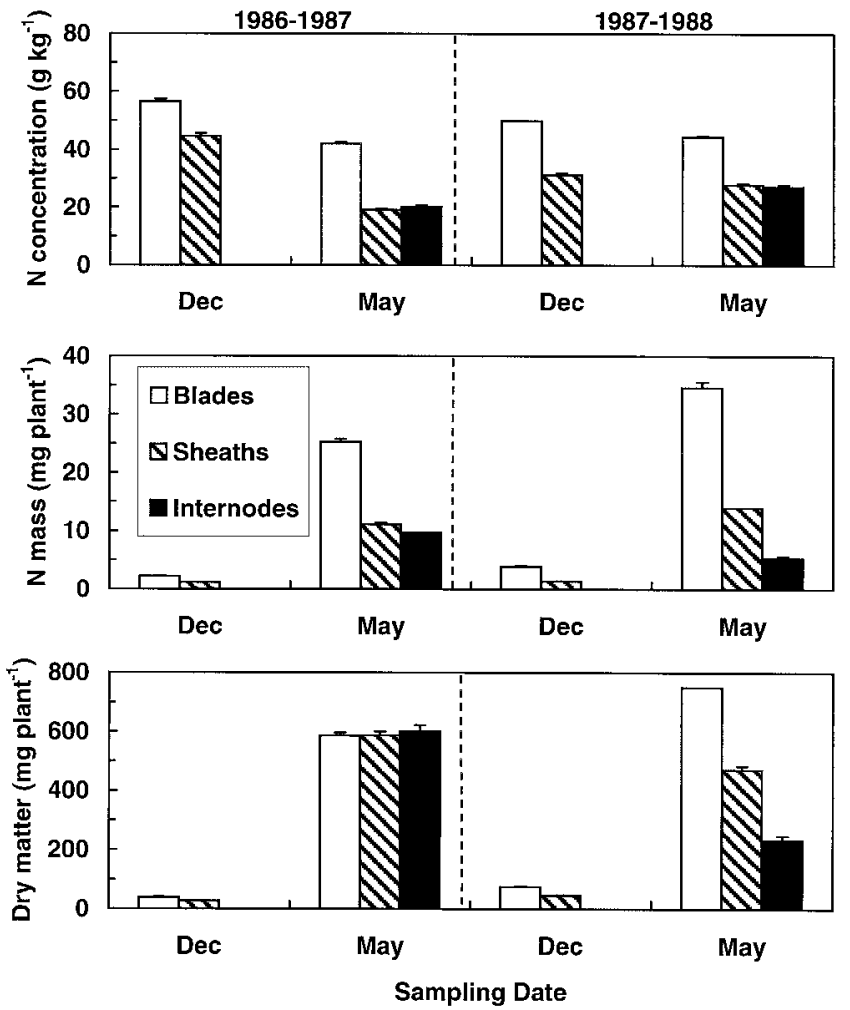

Fig. 1. Blade, sheath, and internode $\mathbf{N}$ concentrations, $\mathbf{N}$ mass, and tissue dry weights on four sampling dates with standard error bars. The MC, T1, T2, and T11 culms are pooled to represent the whole canopy.

cooler $\left(-1.7^{\circ} \mathrm{C}\right)$ and drier $(-51 \mathrm{~mm})$ than average, while the spring and early summer had near-normal temperature $\left(-0.2^{\circ} \mathrm{C}\right)$ and less than average rainfall $(-63 \mathrm{~mm})$. McMaster et al. (1994) gave more detailed weather data.

\section{Whole Canopy}

Most $\mathrm{N}$ and dry weight dynamics studies have used the whole-canopy approach by combining the different plant organs comprising the entire canopy. The MC, T1, T2, and T11 culms comprise the major yield forming culms of winter wheat (McMaster et al., 1994; Power and Alessi, 1978); therefore, we infer whole canopy $\mathrm{N}$ and dry matter dynamics based on pooled values for these culms.

Patterns of $\mathrm{N}$ and dry matter distribution differed between sampling dates and growing seasons (Fig. 1). In December, $\mathrm{N}$ concentration was consistently greater during the first season than in the second season (Table 1 and Fig. 1); in May, $\mathrm{N}$ concentration was slightly greater during the second season (Table 2 and Fig. 1). In December and May, leaf blade $\mathrm{N}$ mass (Fig. 1) and organ weights (Fig. 1 and Tables 3 and 4) were less the first season than the second season. Sheaths and internodes did not show a consistent pattern, but internode weight and $\mathrm{N}$ mass and sheath weight (May only) were lower during the second season. Differences in distribution of dry matter at the May sampling between the first and second season had several causes. First, although sampling occurred at the late jointing stage during both seasons, late jointing is not a precise growth stage (McMaster, 1997). Heat unit accumulation from late winter (1 March) to the May sampling dates was 631 GDD for the first season compared with 482 GDD for the second season. The difference, 149 GDD, is sufficient for complete expansion of about 1.5 internodes (Wilhelm et al., 1993). Indeed, the last two internodes present in the May sampling were larger with lower SE in the first season than second season suggesting that these internodes were closer to completing growth. Another indication of slight differences in growth stage at the May sampling was reflected by the difference in MC Haun stage (i.e., number of leaves produced). The MC Haun was about 10.5 during the first season and 12.5 during the second season. The range in Haun stages associated with the easily observed phenological stage of late jointing amplifies the importance, and complexity, of accurately describing the phe-

Table 1. Weighted mean $\mathbf{N}$ concentration of morphologically identified live culms, phytomers, and tissues (leaf blades and sheaths and internodes) for selected culms of winter wheat in December. Standard errors of the mean are presented following each observation.

\begin{tabular}{|c|c|c|c|c|c|c|c|}
\hline Culm & Phytomer & 1986 & 1987 & Mean & 1986 & 1987 & Mean \\
\hline & & & & & & & \\
\hline \multirow[t]{8}{*}{ MC } & 1 & $56 \pm 2$ & $44 \pm 1$ & $50 \pm 1$ & $20 \pm 2$ & $25 \pm 2$ & $16 \pm 2$ \\
\hline & 2 & $55 \pm 2$ & $54 \pm 1$ & $54 \pm 1$ & $30 \pm 2$ & $17 \pm 2$ & $24 \pm 2$ \\
\hline & 3 & $55 \pm 2$ & $52 \pm 1$ & $53 \pm 1$ & $44 \pm 2$ & $21 \pm 2$ & $33 \pm 2$ \\
\hline & 4 & $58 \pm 2$ & $53 \pm 1$ & $56 \pm 1$ & $67 \pm 2$ & $\mathbf{3 3} \pm \mathbf{3}$ & $50 \pm 2$ \\
\hline & 5 & $69 \pm 8$ & $48 \pm 1$ & $49 \pm 1$ & $79 \pm 8$ & $\mathbf{4 3} \pm \mathbf{3}$ & $44 \pm 3$ \\
\hline & 6 & $\mathbf{N F} \dagger$ & $51 \pm 2$ & $51 \pm 2$ & NF & $52 \pm 3$ & $52 \pm 3$ \\
\hline & 7 & NF & $41 \pm 5$ & $41 \pm 5$ & NF & $54 \pm 10$ & $54 \pm 10$ \\
\hline & Mean & $56 \pm 1$ & $50 \pm 1$ & $53 \pm 1$ & $41 \pm 1$ & $27 \pm 1$ & $33 \pm 1$ \\
\hline \multirow[t]{4}{*}{ T1 } & 1 & $56 \pm 2$ & $53 \pm 1$ & $55 \pm 1$ & $54 \pm 2$ & $25 \pm 2$ & $40 \pm 2$ \\
\hline & 2 & $71 \pm 6$ & $45 \pm 1$ & $48 \pm 1$ & $72 \pm 5$ & $37 \pm \mathbf{3}$ & $41 \pm 2$ \\
\hline & 3 & NF & $45 \pm 2$ & $45 \pm 2$ & NF & $51 \pm 3$ & $51 \pm 3$ \\
\hline & Mean & $58 \pm 2$ & $49 \pm 1$ & $52 \pm 1$ & $56 \pm 2$ & $34 \pm 2$ & $42 \pm 1$ \\
\hline \multirow[t]{4}{*}{$\mathbf{T} 2$} & 1 & $64 \pm 6$ & $52 \pm 2$ & $54 \pm 2$ & $61 \pm 3$ & $\mathbf{3 3} \pm \mathbf{3}$ & $38 \pm 2$ \\
\hline & 2 & NF & $48 \pm 1$ & $48 \pm 1$ & NF & $51 \pm 3$ & $51 \pm 3$ \\
\hline & 3 & NF & $45 \pm 5$ & $45 \pm 5$ & NF & $62 \pm 7$ & $62 \pm 7$ \\
\hline & Mean & $64 \pm 6$ & $50 \pm 1$ & $52 \pm 1$ & $61 \pm 3$ & $41 \pm 2$ & $43 \pm 2$ \\
\hline \multirow[t]{2}{*}{ T11 } & 1 & NF & NF & NF & NF & $53 \pm 6$ & $53 \pm 6$ \\
\hline & Mean & -+ & - & - & - & $53 \pm 6$ & $53 \pm 6$ \\
\hline Grand mean & & $57 \pm 1$ & $\mathbf{5 0} \pm \mathbf{0} \S$ & $52 \pm 0$ & $45 \pm 1$ & $31 \pm \mathbf{1}$ & $36 \pm 1$ \\
\hline
\end{tabular}

$\dagger$ NF, tissue not formed at time of sampling.

+- , calculation not possible.

$\S$ Zero caused by rounding. 
Table 2. Weighted mean $\mathbf{N}$ concentration of morphologically identified live culms, phytomers, and tissues (leaf blades and sheaths and internodes) for selected culms of winter wheat in May. Standard errors of the mean are presented following each observation.

\begin{tabular}{|c|c|c|c|c|c|c|c|c|c|c|}
\hline \multirow[b]{2}{*}{ Culm } & \multirow[b]{2}{*}{ Phytomer } & \multicolumn{3}{|c|}{ Blade } & \multicolumn{3}{|c|}{ Sheath } & \multicolumn{3}{|c|}{ Internode } \\
\hline & & 1986 & 1987 & Mean & 1986 & 1987 & Mean & 1986 & 1987 & Mean \\
\hline & & & & & & g $\mathrm{N} \mathrm{kg}^{-1}$ & & & & \\
\hline \multirow[t]{12}{*}{ MC } & 3 & $\mathbf{M}_{\dagger}$ & $\mathbf{M}$ & $\mathbf{M}$ & $19 \pm 5$ & M & $19 \pm 5$ & $\mathbf{M}$ & $23 \pm 6$ & $23 \pm 6$ \\
\hline & 4 & M & $41 \pm 5$ & $41 \pm 5$ & $18 \pm 5$ & $17 \pm 5$ & $17 \pm 4$ & $17 \pm 7$ & $23 \pm 6$ & $21 \pm 4$ \\
\hline & 5 & M & $37 \pm 2$ & $37 \pm 2$ & $\mathbf{M}$ & $19 \pm 3$ & $19 \pm 3$ & $16 \pm 9$ & $21 \pm 3$ & $20 \pm 3$ \\
\hline & 6 & $40 \pm 3$ & $44 \pm 2$ & $42 \pm 2$ & $17 \pm 2$ & $21 \pm 3$ & $19 \pm 2$ & $14 \pm 3$ & $\mathbf{2 3} \pm \mathbf{2}$ & $19 \pm 2$ \\
\hline & 7 & $36 \pm 1$ & $46 \pm 1$ & $40 \pm 1$ & $17 \pm \mathbf{1}$ & $27 \pm 2$ & $21 \pm 1$ & $14 \pm 3$ & $32 \pm \mathbf{3}$ & $21 \pm 2$ \\
\hline & 8 & $44 \pm 1$ & $48 \pm 2$ & $46 \pm 1$ & $18 \pm 1$ & $29 \pm 2$ & $\mathbf{2 3} \pm \mathbf{1}$ & $\mathbf{1 7} \pm \mathbf{3}$ & $36 \pm \mathbf{3}$ & $22 \pm 3$ \\
\hline & 9 & $45 \pm 1$ & $47 \pm 2$ & $46 \pm 1$ & $20 \pm \mathbf{1}$ & $33 \pm \mathbf{3}$ & $25 \pm 1$ & $19 \pm 3$ & $38 \pm 5$ & $22 \pm 3$ \\
\hline & 10 & $43 \pm 1$ & $49 \pm 2$ & $45 \pm 1$ & $\mathbf{1 8} \pm \mathbf{2}$ & $\mathbf{3 3} \pm \mathbf{3}$ & $22 \pm 1$ & $\mathbf{2 8} \pm \mathbf{3}$ & $37 \pm 6$ & $28 \pm 3$ \\
\hline & 11 & $42 \pm 2$ & $51 \pm 3$ & $43 \pm 1$ & $\mathbf{1 8} \pm \mathbf{2}$ & $33 \pm 4$ & $20 \pm \mathbf{2}$ & $\mathbf{N F}$ & $39 \pm 8$ & $39 \pm 8$ \\
\hline & 12 & NF & $43 \pm 3$ & $43 \pm \mathbf{3}$ & NF & $36 \pm 5$ & $36 \pm 5$ & NF & NF & NF \\
\hline & 13 & NF & $49 \pm 5$ & $49 \pm 5$ & NF & $33 \pm 8$ & $\mathbf{3 3} \pm \mathbf{8}$ & NF & NF & NF \\
\hline & Mean & $42 \pm 1$ & $45 \pm 1$ & $43 \pm 1$ & $18 \pm 1$ & $27 \pm 1$ & $22 \pm 1$ & $18 \pm 1$ & $29 \pm 1$ & $22 \pm 1$ \\
\hline \multirow{9}{*}{ T1 } & 1 & $\mathbf{M}$ & $36 \pm 4$ & $36 \pm 4$ & $\mathbf{M}$ & $15 \pm 5$ & $\mathbf{1 5} \pm 5$ & $16 \pm 9$ & $19 \pm 6$ & $\mathbf{1 7} \pm \mathbf{5}$ \\
\hline & 2 & $\mathbf{M}$ & $42 \pm 3$ & $42 \pm 3$ & $15 \pm 4$ & $19 \pm 2$ & $19 \pm 2$ & $\mathbf{1 8} \pm \mathbf{5}$ & $\mathbf{2 0} \pm \mathbf{3}$ & $19 \pm 3$ \\
\hline & 3 & $39 \pm 2$ & $40 \pm 2$ & $39 \pm 1$ & $16 \pm 3$ & $23 \pm 2$ & $\mathbf{2 0} \pm \mathbf{2}$ & $14 \pm 3$ & $24 \pm 2$ & $17 \pm 2$ \\
\hline & 4 & $41 \pm 1$ & $46 \pm 1$ & $43 \pm 1$ & $17 \pm \mathbf{2}$ & $22 \pm 2$ & $19 \pm 1$ & $15 \pm 3$ & $27 \pm 3$ & $19 \pm 2$ \\
\hline & 5 & $45 \pm 1$ & $47 \pm 1$ & $46 \pm 1$ & $18 \pm 1$ & $28 \pm 2$ & $23 \pm 1$ & $19 \pm 3$ & $37 \pm \mathbf{3}$ & $23 \pm 2$ \\
\hline & 6 & $45 \pm 1$ & $49 \pm 2$ & $46 \pm 1$ & $19 \pm 1$ & $39 \pm 3$ & $26 \pm 1$ & $29 \pm 3$ & $34 \pm 6$ & $30 \pm 3$ \\
\hline & 7 & $43 \pm 2$ & $46 \pm 3$ & $43 \pm 1$ & $24 \pm 1$ & $37 \pm \mathbf{3}$ & $26 \pm 1$ & $35 \pm 5$ & $45 \pm 8$ & $36 \pm 5$ \\
\hline & 8 & $47 \pm 3$ & $47 \pm 4$ & $47 \pm 3$ & $21 \pm 2$ & $43 \pm 5$ & $\mathbf{2 4} \pm \mathbf{2}$ & NF & NF & NF \\
\hline & Mean & $43 \pm 1$ & $45 \pm 1$ & $44 \pm 1$ & $19 \pm 1$ & $26 \pm 1$ & $22 \pm \mathbf{1}$ & $\mathbf{2 0} \pm \mathbf{1}$ & $\mathbf{2 5} \pm \mathbf{1}$ & $22 \pm 1$ \\
\hline \multirow[t]{9}{*}{$\mathbf{T 2}$} & 1 & $\mathbf{M}$ & $36 \pm 4$ & $36 \pm 4$ & $16 \pm 5$ & $16 \pm 5$ & $16 \pm 4$ & $21 \pm 5$ & $\mathbf{2 3} \pm \mathbf{5}$ & $22 \pm 4$ \\
\hline & 2 & $36 \pm 3$ & $36 \pm 2$ & $36 \pm 2$ & $\mathbf{1 7} \pm \mathbf{3}$ & $21 \pm 3$ & $19 \pm 2$ & $17 \pm 4$ & $\mathbf{1 8} \pm \mathbf{3}$ & $18 \pm 2$ \\
\hline & 3 & $40 \pm 2$ & $44 \pm 1$ & $42 \pm 1$ & $\mathbf{1 8} \pm \mathbf{2}$ & $25 \pm 3$ & $21 \pm 1$ & $15 \pm 3$ & $\mathbf{2 8} \pm \mathbf{3}$ & $19 \pm 2$ \\
\hline & 4 & $44 \pm 1$ & $48 \pm 1$ & $46 \pm 1$ & $\mathbf{1 7} \pm \mathbf{1}$ & $30 \pm 2$ & $21 \pm 1$ & $81 \pm \mathbf{3}$ & $26 \pm 4$ & $20 \pm 2$ \\
\hline & 5 & $47 \pm 1$ & $49 \pm 1$ & $48 \pm 1$ & $20 \pm 1$ & $35 \pm 2$ & $26 \pm 1$ & $25 \pm 3$ & $34 \pm 3$ & $27 \pm 2$ \\
\hline & 6 & $44 \pm 1$ & $47 \pm 2$ & $45 \pm 1$ & $20 \pm \mathbf{2}$ & $43 \pm 3$ & $27 \pm 1$ & $33 \pm 4$ & $60 \pm 8$ & $34 \pm 4$ \\
\hline & 7 & $42 \pm 2$ & $43 \pm 3$ & $43 \pm 2$ & $20 \pm \mathbf{2}$ & $42 \pm 4$ & $24 \pm 2$ & NF & $30 \pm 8$ & $30 \pm 8$ \\
\hline & 8 & $52 \pm 5$ & $48 \pm 5$ & $51 \pm 4$ & $21 \pm 4$ & $35 \pm 8$ & $\mathbf{2 3} \pm \mathbf{3}$ & NF & NF & NF \\
\hline & Mean & $43 \pm 1$ & $45 \pm 1$ & $44 \pm 1$ & $19 \pm 1$ & $28 \pm 1$ & $\mathbf{2 3} \pm \mathbf{1}$ & $22 \pm 1$ & $26 \pm 2$ & $23 \pm 1$ \\
\hline \multirow[t]{6}{*}{ T11 } & 1 & $32 \pm 2$ & $35 \pm 2$ & $34 \pm 1$ & $20 \pm \mathbf{2}$ & $28 \pm 3$ & $24 \pm 2$ & $25 \pm 7$ & $\mathbf{5 0} \pm \mathbf{8}$ & $\mathbf{3 7} \pm \mathbf{5}$ \\
\hline & 2 & $37 \pm 2$ & $41 \pm 2$ & $39 \pm 1$ & $29 \pm 2$ & $34 \pm 2$ & $32 \pm 2$ & M & $22 \pm 8$ & $22 \pm 8$ \\
\hline & 3 & $45 \pm 3$ & $47 \pm 2$ & $46 \pm 2$ & $32 \pm 2$ & $35 \pm 3$ & $34 \pm 2$ & $60 \pm 9$ & $39 \pm 8$ & $44 \pm 7$ \\
\hline & 4 & M & $49 \pm 2$ & $49 \pm 2$ & $29 \pm 4$ & $41 \pm 3$ & $39 \pm 3$ & $45 \pm 9$ & $39 \pm 8$ & $42 \pm 6$ \\
\hline & 5 & $48 \pm 5$ & $50 \pm 4$ & $\mathbf{5 0} \pm \mathbf{3}$ & $80 \pm 5$ & $36 \pm 6$ & $45 \pm 5$ & NF & NF & NF \\
\hline & Mean & $38 \pm 1$ & $42 \pm 1$ & $40 \pm 1$ & $27 \pm 1$ & $33 \pm 1$ & $31 \pm 1$ & $34 \pm 5$ & $36 \pm 4$ & $35 \pm 3$ \\
\hline Grand mean & & $42 \pm 0 \S$ & $44 \pm 0$ & $43 \pm 0$ & $19 \pm 0$ & $28 \pm 1$ & $23 \pm 0$ & $\mathbf{2 0} \pm \mathbf{1}$ & $27 \pm 1$ & $22 \pm 1$ \\
\hline
\end{tabular}

$\dagger \mathrm{M}$, tissue was missing or not identifiable at time of sampling (usually older tissues). $\$$ NF, tissue not formed at time of sampling

$\S$ Zero caused by rounding.

Table 3. Dry weight of morphologically identified live culms, phytomers, and tissues (leaf blades and sheaths and internodes) for selected culms of winter wheat in December. Standard errors of the mean are presented following each observation.

\begin{tabular}{|c|c|c|c|c|c|c|c|}
\hline \multirow[b]{2}{*}{ Culm } & \multirow[b]{2}{*}{ Phytomer } & \multicolumn{3}{|c|}{ Blade } & \multicolumn{3}{|c|}{ Sheath } \\
\hline & & 1985 & 1987 & Mean & 1986 & 1987 & Mean \\
\hline \multirow{9}{*}{ MC } & & & & & & & \\
\hline & 1 & $8 \pm 1$ & $5 \pm 1$ & $7 \pm 1$ & $4 \pm 0 \S$ & $4 \pm 1$ & $4 \pm 0$ \\
\hline & 2 & $11 \pm 1$ & $\mathbf{8} \pm \mathbf{1}$ & $10 \pm \mathbf{1}$ & $6 \pm 0$ & $4 \pm 1$ & $\mathbf{5} \pm \mathbf{0}$ \\
\hline & 3 & $13 \pm 1$ & $\mathbf{1 3} \pm \mathbf{1}$ & $13 \pm 1$ & $6 \pm 1$ & $6 \pm 1$ & $6 \pm 0$ \\
\hline & 4 & $4 \pm 1$ & $16 \pm 1$ & $\mathbf{1 0} \pm \mathbf{1}$ & $6 \pm 0$ & $7 \pm \mathbf{1}$ & $6 \pm 0$ \\
\hline & 5 & $3 \pm 3$ & $14 \pm 1$ & $14 \pm 1$ & $3 \pm 1$ & $7 \pm 1$ & $6 \pm \mathbf{1}$ \\
\hline & 6 & $\mathbf{N F} \dagger$ & $6 \pm 1$ & $6 \pm 1$ & NF & $6 \pm 1$ & $\mathbf{6} \pm \mathbf{1}$ \\
\hline & 7 & NF & $2 \pm 4$ & $2 \pm 4$ & NF & $6 \pm 2$ & $6 \pm 2$ \\
\hline & Total & $35 \pm 1$ & $53 \pm 2$ & $44 \pm 1$ & $22 \pm 1$ & $27 \pm 1$ & $24 \pm 1$ \\
\hline \multirow[t]{4}{*}{ T1 } & 1 & $4 \pm 1$ & $6 \pm 1$ & $\mathbf{5} \pm \mathbf{1}$ & $6 \pm 0$ & $6 \pm 1$ & $6 \pm 0$ \\
\hline & 2 & $1 \pm 2$ & $7 \pm 1$ & $6 \pm 1$ & $4 \pm 1$ & $6 \pm 1$ & $5 \pm \mathbf{0}$ \\
\hline & 3 & NF & $5 \pm 1$ & $5 \pm 1$ & NF & $6 \pm 1$ & $6 \pm 1$ \\
\hline & Total & $4 \pm 1$ & $13 \pm 1$ & $8 \pm 1$ & $6 \pm 0$ & $12 \pm 1$ & $7 \pm 0$ \\
\hline \multirow[t]{4}{*}{$\mathbf{T} 2$} & 1 & $1 \pm 2$ & $\mathbf{8} \pm \mathbf{1}$ & $7 \pm 1$ & $4 \pm 0$ & $6 \pm 1$ & $\mathbf{6} \pm \mathbf{0}$ \\
\hline & 2 & NF & $6 \pm 1$ & $6 \pm 1$ & NF & $5 \pm 1$ & $5 \pm \mathbf{1}$ \\
\hline & 3 & NF & $3 \pm 4$ & $3 \pm 4$ & NF & $6 \pm 1$ & $\mathbf{6} \pm \mathbf{1}$ \\
\hline & Total & $\mathbf{0} \pm \mathbf{0}$ & $\mathbf{1 0} \pm \mathbf{1}$ & $5 \pm 1$ & $\mathbf{1} \pm \mathbf{0}$ & $8 \pm 1$ & $4 \pm 0$ \\
\hline \multirow[t]{2}{*}{ T11 } & 1 & NF & NF & NF & NF & $5 \pm 1$ & $5 \pm 1$ \\
\hline & Total & $-\ddagger$ & - & - & - & $\mathbf{0} \pm \mathbf{0}$ & $\mathbf{0} \pm \mathbf{0}$ \\
\hline Grand total & & $39 \pm 2$ & $75 \pm 3$ & $57 \pm 2$ & $28 \pm 1$ & $46 \pm 1$ & $35 \pm 1$ \\
\hline
\end{tabular}

$\dagger$ NF, tissue not formed at time of sampling. +- , calculation not possible.

$\S$ Zero caused by rounding. 
Table 4. Dry weight of morphologically identified live culms, phytomers, and tissues (leaf blades and sheaths and internodes) for selected culms of winter wheat in May. Standard errors of the mean are presented following each observation.

\begin{tabular}{|c|c|c|c|c|c|c|c|c|c|c|}
\hline \multirow[b]{2}{*}{ Culm } & \multirow[b]{2}{*}{ Phytomer } & \multicolumn{3}{|c|}{ Blade } & \multicolumn{3}{|c|}{ Sheath } & \multicolumn{3}{|c|}{ Internode } \\
\hline & & 1986 & 1987 & Mean & 1986 & 1987 & Mean & 1986 & 1987 & Mean \\
\hline & & & & & & mg & & & & \\
\hline \multirow[t]{12}{*}{ MC } & 3 & $\mathbf{M} \dagger$ & M & $\mathbf{M}$ & $3 \pm 1$ & M & $3 \pm 1$ & M & $5 \pm 14$ & $5 \pm 14$ \\
\hline & 4 & M & $75 \pm 13$ & $75 \pm 13$ & $7 \pm 1$ & $22 \pm 6$ & $19 \pm 5$ & $3 \pm 13$ & $29 \pm 14$ & $20 \pm 10$ \\
\hline & 5 & M & $28 \pm 8$ & $28 \pm 8$ & $\mathbf{M}$ & $13 \pm 5$ & $13 \pm 5$ & $21 \pm 19$ & $28 \pm 6$ & $26 \pm 7$ \\
\hline & 6 & $36 \pm 5$ & $\mathbf{5 0} \pm \mathbf{5}$ & $44 \pm 4$ & $16 \pm 5$ & $31 \pm 4$ & $\mathbf{2 4} \pm \mathbf{3}$ & $47 \pm 6$ & $40 \pm 6$ & $44 \pm 4$ \\
\hline & 7 & $42 \pm 2$ & $65 \pm 5$ & $52 \pm 3$ & $27 \pm \mathbf{3}$ & $37 \pm 3$ & $31 \pm 2$ & $76 \pm 6$ & $31 \pm 6$ & $58 \pm 4$ \\
\hline & 8 & $52 \pm 2$ & $74 \pm 5$ & $61 \pm 3$ & $44 \pm 3$ & $47 \pm 3$ & $45 \pm 2$ & $75 \pm 6$ & $31 \pm 6$ & $63 \pm 4$ \\
\hline & 9 & $58 \pm 2$ & $66 \pm 5$ & $61 \pm 3$ & $57 \pm \mathbf{3}$ & $47 \pm 4$ & $\mathbf{5 3} \pm \mathbf{3}$ & $59 \pm 6$ & $21 \pm 12$ & $54 \pm 5$ \\
\hline & 10 & $\mathbf{5 3} \pm \mathbf{2}$ & $72 \pm 8$ & $\mathbf{5 8} \pm \mathbf{3}$ & $\mathbf{7 3} \pm \mathbf{3}$ & $52 \pm 4$ & $67 \pm 3$ & $36 \pm 7$ & $4 \pm 14$ & $34 \pm 6$ \\
\hline & 11 & $41 \pm 3$ & $84 \pm 11$ & $47 \pm 3$ & $84 \pm 4$ & $54 \pm 6$ & $80 \pm \mathbf{3}$ & $\mathbf{N F} \div$ & $7 \pm 20$ & $7 \pm 20$ \\
\hline & 12 & NF & $61 \pm 11$ & $61 \pm 11$ & NF & $54 \pm 7$ & $54 \pm 7$ & NF & $\mathbf{N F}$ & $\mathbf{N F}$ \\
\hline & 13 & NF & $27 \pm 19$ & $37 \pm 19$ & NF & $27 \pm 13$ & $27 \pm 13$ & NF & NF & NF \\
\hline & Total & $238 \pm 5$ & $297 \pm 11$ & $264 \pm 5$ & $243 \pm 7$ & $184 \pm 7$ & $217 \pm 5$ & $270 \pm 12$ & $97 \pm 9$ & $194 \pm 8$ \\
\hline \multirow{9}{*}{ T1 } & 1 & M & $6 \pm 13$ & $6 \pm 13$ & $\mathbf{M}$ & $5 \pm 7$ & $5 \pm 7$ & $9 \pm 19$ & $5 \pm 14$ & $7 \pm 12$ \\
\hline & 2 & M & $22 \pm 9$ & $22 \pm 9$ & $9 \pm 8$ & $9 \pm 4$ & $9 \pm 3$ & $34 \pm 10$ & $15 \pm 8$ & $20 \pm 7$ \\
\hline & 3 & $33 \pm 4$ & $36 \pm 5$ & $35 \pm 3$ & $15 \pm 6$ & $18 \pm 4$ & $17 \pm 3$ & $53 \pm 6$ & $47 \pm 6$ & $50 \pm 5$ \\
\hline & 4 & $39 \pm 2$ & $63 \pm 5$ & $\mathbf{5 0} \pm \mathbf{3}$ & $29 \pm 4$ & $34 \pm 3$ & $31 \pm 2$ & $58 \pm 6$ & $32 \pm 7$ & $48 \pm 4$ \\
\hline & 5 & $48 \pm 2$ & $73 \pm 5$ & $59 \pm \mathbf{3}$ & $39 \pm 3$ & $44 \pm 3$ & $41 \pm 2$ & $54 \pm 6$ & $18 \pm 7$ & $46 \pm 5$ \\
\hline & 6 & $47 \pm 2$ & $57 \pm 6$ & $51 \pm \mathbf{3}$ & $48 \pm \mathbf{3}$ & $48 \pm 4$ & $48 \pm 3$ & $30 \pm 6$ & $10 \pm 12$ & $29 \pm 5$ \\
\hline & 7 & $39 \pm 2$ & $83 \pm 8$ & $46 \pm 2$ & $59 \pm 3$ & $53 \pm 5$ & $\mathbf{5 8} \pm \mathbf{3}$ & $22 \pm 11$ & $4 \pm 20$ & $20 \pm 10$ \\
\hline & 8 & $39 \pm 6$ & $69 \pm 13$ & $44 \pm 5$ & $83 \pm 4$ & $63 \pm 7$ & $79 \pm 4$ & $\mathbf{N F}$ & $\mathbf{N F}$ & NF \\
\hline & Total & $190 \pm 5$ & $235 \pm 11$ & $209 \pm 6$ & $189 \pm 7$ & $142 \pm 7$ & $168 \pm 5$ & $181 \pm 11$ & $70 \pm 8$ & $132 \pm 7$ \\
\hline \multirow{9}{*}{$\mathbf{T 2}$} & 1 & M & $10 \pm \mathbf{1 3}$ & $10 \pm 13$ & $8 \pm 11$ & $4 \pm 7$ & $4 \pm 7$ & $7 \pm \mathbf{1 1}$ & $5 \pm 12$ & $6 \pm 8$ \\
\hline & 2 & $11 \pm 5$ & $26 \pm 7$ & $20 \pm 4$ & $7 \pm 6$ & $7 \pm 5$ & $7 \pm 4$ & $17 \pm 7$ & $35 \pm 7$ & $26 \pm 5$ \\
\hline & 3 & $29 \pm 3$ & $39 \pm 5$ & $34 \pm 3$ & $14 \pm 4$ & $24 \pm 4$ & $18 \pm 3$ & $46 \pm 6$ & $34 \pm 6$ & $41 \pm 4$ \\
\hline & 4 & $37 \pm 2$ & $51 \pm 5$ & $43 \pm 2$ & $26 \pm 3$ & $36 \pm 3$ & $31 \pm 2$ & $56 \pm 6$ & $30 \pm 9$ & $49 \pm 4$ \\
\hline & 5 & $42 \pm 2$ & $58 \pm 5$ & $49 \pm 3$ & $\mathbf{3 8} \pm \mathbf{3}$ & $45 \pm 4$ & $41 \pm 2$ & $36 \pm 6$ & $17 \pm 8$ & $33 \pm 5$ \\
\hline & 6 & $38 \pm 2$ & $49 \pm 7$ & $42 \pm 3$ & $\mathbf{5 3} \pm \mathbf{3}$ & $40 \pm 5$ & $49 \pm 3$ & $18 \pm 8$ & $5 \pm 20$ & $17 \pm 8$ \\
\hline & 7 & $32 \pm \mathbf{3}$ & $23 \pm 11$ & $\mathbf{3 1} \pm \mathbf{3}$ & $65 \pm 4$ & $43 \pm 6$ & $62 \pm 3$ & NF & $4 \pm 20$ & $4 \pm 20$ \\
\hline & 8 & $38 \pm 8$ & $57 \pm 19$ & $41 \pm 7$ & $81 \pm 8$ & $63 \pm 13$ & $78 \pm 7$ & NF & NF & NF \\
\hline & Total & $151 \pm 5$ & $185 \pm 10$ & $166 \pm 5$ & $151 \pm 7$ & $126 \pm 8$ & $140 \pm 5$ & $150 \pm 11$ & $64 \pm 8$ & $112 \pm 7$ \\
\hline \multirow[t]{6}{*}{ T11 } & 1 & $8 \pm \mathbf{3}$ & $8 \pm 6$ & $8 \pm 4$ & $4 \pm 4$ & $5 \pm 5$ & $5 \pm 3$ & $5 \pm \mathbf{1 4}$ & $2 \pm 20$ & $4 \pm 12$ \\
\hline & 2 & $8 \pm \mathbf{3}$ & $11 \pm 7$ & $10 \pm 4$ & $5 \pm 4$ & $8 \pm 4$ & $6 \pm 3$ & $\mathbf{M}$ & $6 \pm 20$ & $6 \pm 20$ \\
\hline & 3 & $4 \pm 5$ & $31 \pm 7$ & $19 \pm 4$ & $5 \pm 5$ & $11 \pm 5$ & $9 \pm 3$ & $2 \pm 19$ & $7 \pm 20$ & $6 \pm 16$ \\
\hline & 4 & M & $38 \pm 7$ & $38 \pm 7$ & $6 \pm 11$ & $23 \pm 5$ & $21 \pm 5$ & $2 \pm 19$ & $6 \pm 20$ & $4 \pm 14$ \\
\hline & 5 & $10 \pm 8$ & $24 \pm 13$ & $21 \pm 11$ & $8 \pm 11$ & $40 \pm 9$ & $33 \pm 8$ & $\mathbf{N F}$ & NF & NF \\
\hline & Total & $8 \pm 2$ & $33 \pm 7$ & $19 \pm 3$ & $5 \pm 3$ & $20 \pm 4$ & $12 \pm 2$ & $0 \pm 1$ & $1 \pm \mathbf{3}$ & $1 \pm 2$ \\
\hline Grand total & & $587 \pm 9$ & $750 \pm 20$ & $\mathbf{6 5 8} \pm \mathbf{1 0}$ & $587 \pm \mathbf{1 3}$ & $472 \pm 13$ & $537 \pm 9$ & $602 \pm 20$ & $233 \pm 15$ & $440 \pm 13$ \\
\hline
\end{tabular}

$\dagger M$, tissue was missing or not identifiable at time of sampling (usually older tissues).

$\$$ NF, tissue not formed at time of sampling.

nostage of a crop and how descriptions on one scale may differ in meaning on another scale.

Interestingly, during the first season, fewer leaves appeared at the time of the May sampling than during the second season (11 vs. 13). In addition, more of the older leaves (in the lower part of the canopy) had also senesced in the first season; leaves 1 to 5 in the first season compared to leaves 1 to 3 in the second season (note the phytomer data represented by "M," indicating the organs were missing at the time of sampling in Tables 1 to 4). In addition to having more leaves on culms in the second season (the drier of the two seasons), the leaf blades were larger, but sheaths and internodes were smaller (Tables 3 and 4). Certainly water stress reduces plant height (Blum and Sullivan, 1997; Ehdaie, 1995), and it could be hypothesized that during water stress it is better to sacrifice plant height (i.e., sheath and internode length) than primary photosynthetic tissue (i.e., blades).

When pooled across seasons, $\mathrm{N}$ concentration decreased in all live tissues from the December to May sampling; blades went from about $52 \mathrm{~g} \mathrm{~N} \mathrm{~kg}^{-1}$ in December to $43 \mathrm{~g} \mathrm{~N} \mathrm{~kg}^{-1}$ in May, sheaths went from $36 \mathrm{~g} \mathrm{~N}$ $\mathrm{kg}^{-1}$ in December to $23 \mathrm{~g} \mathrm{~N} \mathrm{~kg}^{-1}$ in May, while internodes, which were not present in December, were $22 \mathrm{~g}$
$\mathrm{N} \mathrm{kg}^{-1}$ in May (Table 2). Sheaths and internodes had similar concentrations in May (Fig. 2). Nitrogen mass and dry weight increased from December to May for all plant organs and was greatest for blades and least for internodes (Fig. 2). Mean $\mathrm{N}$ mass was correlated more closely with mean tissue weight $(r=0.89, p<$ $0.001, n=29)$ than $\mathrm{N}$ concentration $(r=-0.10, p>$ $0.5, n=29)$.

\section{Individual Culms}

Nitrogen and dry weight dynamics for individual culms were similar to the whole canopy (Fig. 3; Tables 1-4). As with whole canopies, $\mathrm{N}$ concentration was greater and $\mathrm{N}$ mass and dry weight was lower in December than May for blades and sheaths of all culms. Mean $\mathrm{N}$ concentration of all tissues on a culm ranged from about $43 \mathrm{~g} \mathrm{~N} \mathrm{~kg}^{-1}$ for $\mathrm{MC}$ to $48 \mathrm{~g} \mathrm{~N} \mathrm{~kg}^{-1}$ for $\mathrm{T} 2$ in December and from about $29 \mathrm{~g} \mathrm{~N} \mathrm{~kg}^{-1}$ for MC to $35 \mathrm{~g} \mathrm{~N}$ $\mathrm{kg}^{-1}$ for T11 in May. These changes in $\mathrm{N}$ concentration reflect the order of culm appearance $(\mathrm{MC}>\mathrm{T} 1>\mathrm{T} 2>$ T11; Klepper et al., 1983), and therefore age, of the culms. Mean MC N concentrations were $53 \mathrm{~g} \mathrm{~N} \mathrm{~kg}^{-1}$ in December (early tillering) and $43 \mathrm{~g} \mathrm{~N} \mathrm{~kg}^{-1}$ in May (late 

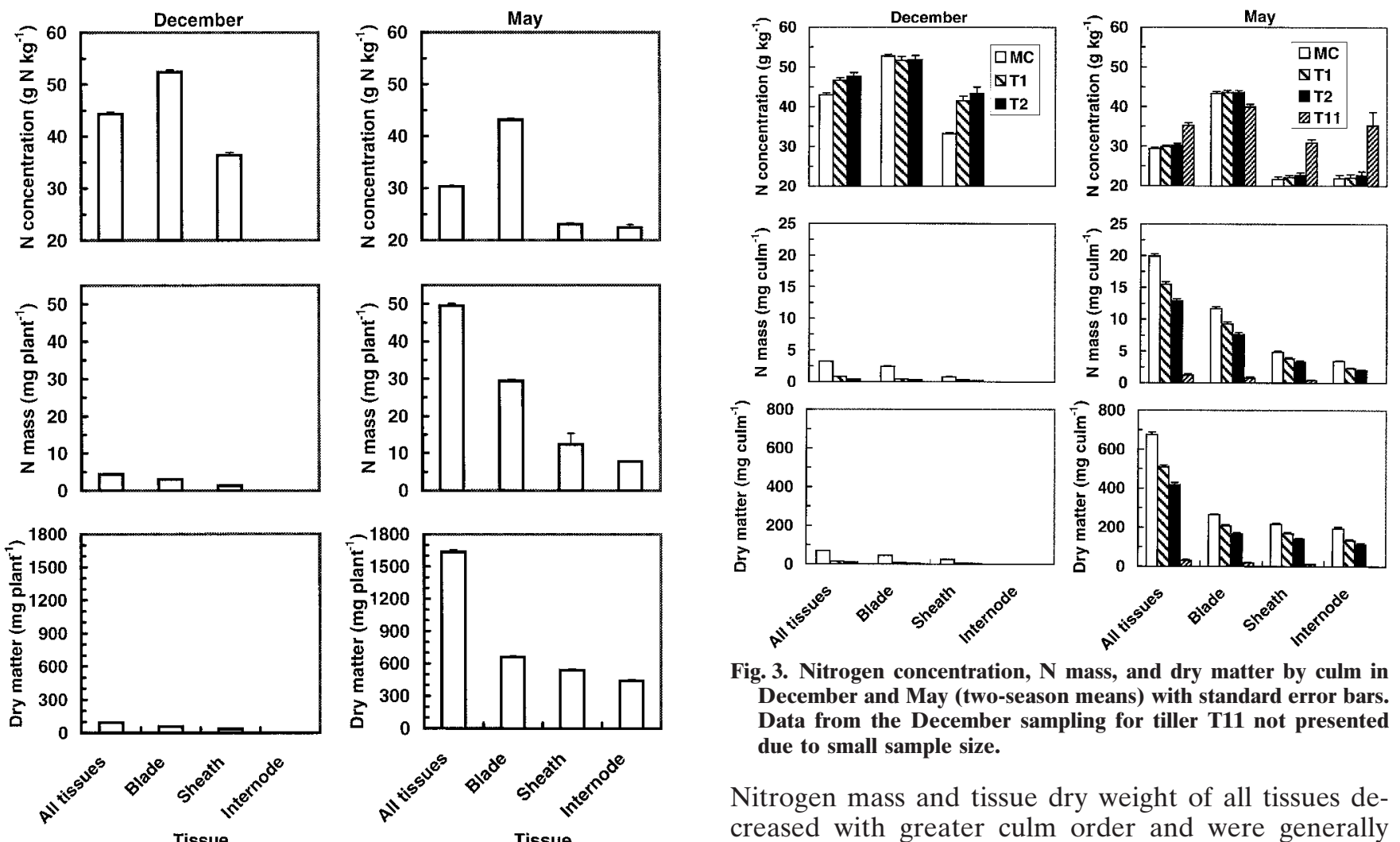

Fig. 3. Nitrogen concentration, $\mathbf{N}$ mass, and dry matter by culm in December and May (two-season means) with standard error bars. Data from the December sampling for tiller T11 not presented due to small sample size.

Nitrogen mass and tissue dry weight of all tissues decreased with greater culm order and were generally greater for blades and less for sheaths and internodes. It is interesting to note the similarity between sheath and internode $\mathrm{N}$ concentrations on all culms in May, indicating that the standard sampling technique of including sheaths with stem tissue does not alter the estimate of stem $\mathrm{N}$ concentration.

\section{Phytomer Position within the Canopy}

A phytomer is defined as a repeating structural unit of a plant comprised of a leaf, node-internode, and axillary bud (Wilhelm and McMaster, 1995). The first phytomer to appear on a culm is designated Position 1; numbering of successive phytomers increases acropetally on each culm. One can view a grass sward or canopy as a population of phytomers distributed among different culms (Harper, 1977). Taking this perspective we will first contrast phytomer positions, regardless of culm, to examine the general pattern of $\mathrm{N}$ and dry matter distribution among these structural units.

Phytomer (leaf) position was a significant $(p<0.05)$ source of variation on all sampling dates, for all tissues, and all traits, except for the May 1988 internode $\mathrm{N}$ mass and tissue weight $(p>0.20)$. Examination of the $\mathrm{N}$ concentration data by phytomer position (Fig. 4) reveals that older phytomers (lower numbers) have lower concentrations. Nitrogen concentration appeared to approach a relatively constant lower limit as tissues aged, but that lower limit differed for December and May. In December, the three oldest phytomers had $\mathrm{N}$ concentrations of about $43 \mathrm{~g} \mathrm{~N} \mathrm{~kg}^{-1}$, while $\mathrm{N}$ concentration for younger phytomers ranged from 46 to $53 \mathrm{~g} \mathrm{~N} \mathrm{~kg}^{-1}$. In May, Phytomers 1 to 4 had $\mathrm{N}$ concentrations around $28 \mathrm{~g} \mathrm{~N} \mathrm{~kg}^{-1}$, while Phytomers 5 to 11 had concentrations 

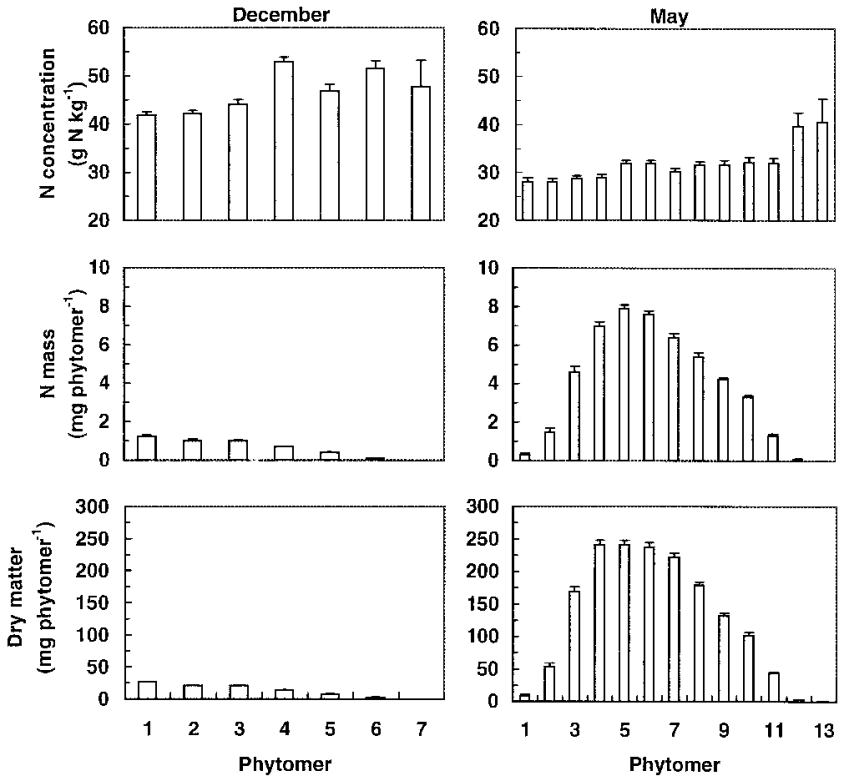

Fig. 4. Nitrogen concentration, $\mathbf{N}$ mass, and dry matter by phytomer (two-season means over all tissues and culms) with standard error bars.

near $32 \mathrm{~g} \mathrm{~N} \mathrm{~kg}^{-1}$. Only the very youngest tissues (Phytomers 12 and 13) had appreciably greater $\mathrm{N}$ concentrations, around $40 \mathrm{~g} \mathrm{~N} \mathrm{~kg}^{-1}$. These data support the assumption used in development of the SHOOTGRO model (Wilhelm et al., 1993); new tissue on a wheat plant is produced with an $\mathrm{N}$ concentration of $40 \mathrm{~g} \mathrm{~N}$ $\mathrm{kg}^{-1}$. However, the assumption that senesced tissue declines to an $\mathrm{N}$ concentration of $10 \mathrm{~g} \mathrm{~N} \mathrm{~kg}^{-1}$ is not consistent with data collected in this study. Senesced tissue had an $\mathrm{N}$ concentration of $19( \pm 1) \mathrm{g} \mathrm{N} \mathrm{kg}^{-1}$.

The size of fully developed organs tends to be greater on later-produced phytomers (Gallagher, 1979; Hay and Wilson, 1982; Kirby, 1988; Fig. 4). In December, dry matter was $26.3 \mathrm{mg}$ for Phytomer 1 and $8 \mathrm{mg}$ for Phytomer 5, the youngest phytomer present in December of both seasons. Only Phytomers 1 and 2 were fully mature at the December sampling, explaining the apparent contradiction between the previous two sentences. A similar difference occurred in $\mathrm{N}$ mass (difference of $\approx 1$ $\mathrm{mg}$ ). In May, older phytomers (1-3) had less $\mathrm{N}$ mass and dry matter than later-produced phytomers (younger) that had reached mature size (4-7). These data may also reflect some of the impact of tissue senescence, in that the older phytomers may have translocated some $\mathrm{N}$ and assimilate to younger, developing tissues. The youngest phytomers (8-13, still growing at the time of sampling) had lower dry matter and $\mathrm{N}$ mass than the mature phytomers (4-7). These patterns reflect the distribution of organ size within the plant population. Higher-numbered (younger) phytomers had a smaller fraction of the total $\mathrm{N}$ mass and dry weight because fewer culms had developed those phytomers, and the tissues on highernumbered phytomers were more likely to be the immature (not yet full-size) tissues on the culms that bore them.

\section{Phytomer Position on Individual Culms}

Another perspective for examining phytomer $\mathrm{N}$ and dry weight dynamics is to examine phytomer positions within a specific culm. As indicated above, younger phytomers (those with higher numbers), on all culms, had greater $\mathrm{N}$ concentrations than older phytomers and concentrations were greater in December than May (Fig. 4). Presumably this pattern is due to new tissues growing at greater $\mathrm{N}$ concentrations before dilution of $\mathrm{N}$ by the addition of assimilates during growth and subsequent translocation of $\mathrm{N}$ away from older, fully grown tissue to developing tissue.

With the small number of phytomer positions to compare among culms in December because of the young age of tillers, we will discuss only the May sampling to compare phytomer positions for the remainder of this section (Table 5). The most discernable pattern for phytomer positions across culms is the similarity of $\mathrm{N}$ concentrations. However, a tendency was seen for specific phytomers on the MC to have lower $\mathrm{N}$ concentrations than those phytomers on T1 or T2, while phytomers on T11 had the greatest N concentration. Interestingly, since $\mathrm{N}$ taken up by the roots must initially flow through the vascular tissue of the MC to reach primary and secondary tillers (because nodal roots are considerably less developed on tillers than the MC at the May sampling; McMaster, 1997), traditional thinking would suggest the inverse pattern should be observed due to a hierarchy for access to $\mathrm{N}$ (Thorne, 1982). Our data do not support this assumption, but rather appear to conform to the concept expressed by Skinner et al. (1999) that $\mathrm{N}$ cycles throughout the plant before finally being utilized in specific tissue. Alternatively, our observation may be just another manifestation of tissue age because Phytomer 1 on MC is older, by at least two phyllochrons (Klepper et al., 1982), than Phytomer 1 on T1; likewise, both are older than Phytomer 1 on T11 and older tissues have lesser $\mathrm{N}$ concentrations than younger tissues.

Dry weight of mature phytomers within a culm, especially tillers, did seem to increase with later appearance (higher numbers), but this pattern was clouded due to older phytomers often either having no internodes (they did not elongate) or senesced blade and sheath tissue and younger phytomers having currently elongating internodes, blades, and sheaths (Table 5). When comparing dry weights of a specific phytomer (all tissues) across culms, dry weight usually decreased from $\mathrm{T} 1$ to $\mathrm{T} 2$ to MC to T11. Phytomers 7 and 8 of the MC were exceptions to this generalization because internode growth on the tillers at these positions had not yet started, or just begun (Table 5, blade plus sheath dry weights were very similar to total dry weights). Since main culms generally are thought to be bigger and taller than tillers (McMaster, 1997; Power and Alessi, 1978), it was surprising to find mass of phytomers on the MC less than on primary tillers. Indeed, the reason that main culms seem taller at late jointing, is that more internodes have begun elongating. In addition, main culms may seem larger because more leaves are present (both produced and remaining) on the culm (Tables 1,2, and 5). For similar reasons, T11 is shorter and smaller because fewer 
Table 5. Weighted mean $\mathbf{N}$ concentration and dry weights of morphologically identified phytomers for selected culms of winter wheat. Data for both seasons are pooled. Standard errors presented in Tables $\mathbf{1}$ and 2.

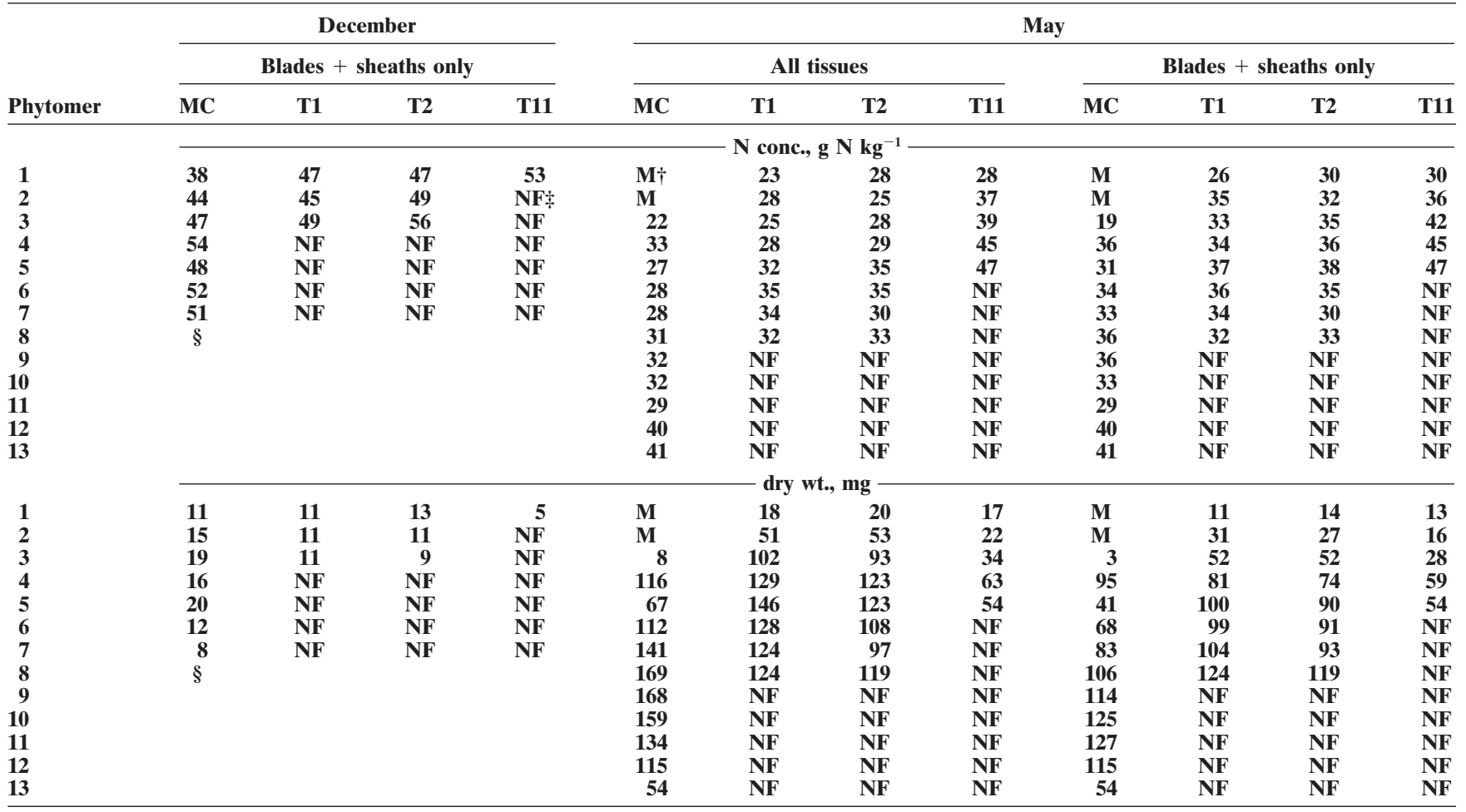

$\dagger \mathrm{M}$, tissue was missing or not identifiable at times of sampling (usually older tissues).

$\$$ NF, tissue not formed at time of sampling.

$\S$ No more than seven phytomers were formed on any culm at the December sampling.

internodes elongate and fewer leaves are produced. While blade, sheath, and internode size is clearly inversely correlated with phytomer position, at least up to some high number (Hay and Wilson, 1982; McMaster, 1997), when pooling a phytomer position for a specific culm across different plants in a field, our data do not support the hypothesis that main culms are able to exploit their competitive advantage (e.g., taller, more leaves, more nodal roots plus seminal roots) and produce larger phytomers than primary tillers. Rather, the apparent differences between main culm and primary tillers, when considered as a population of phytomers on a field scale, is due to earlier development of internodes and leaves, and slower leaf senescence.

\section{CONCLUSIONS}

Many similar patterns of $\mathrm{N}$ dynamics and dry weight distributions were observed for different organizational levels of the wheat canopy during vegetative development. At the whole-canopy level, our results were similar to previously published work; as the canopy developed with older tissue contributing more of the total tissue, total canopy $\mathrm{N}$ concentration decreased even though $\mathrm{N}$ mass increased because dry matter (mass) increased faster than $\mathrm{N}$ mass as a result of canopy growth. Examining at a finer level of resolution, dry matter and $\mathrm{N}$ mass decreased from MC to primary Tillers $\mathrm{T} 1$ and $\mathrm{T} 2$ and was least for secondary Tiller T11, while the inverse relationship was found for $\mathrm{N}$ concentration. The reason for these relationships became clearer when considering the fundamental building block of a culm, the phytomer. Young culms had less dry weight and $\mathrm{N}$ mass than earlier appearing culms because recently appearing culms had phytomers that were smaller (not completely expanded) and fewer of these phytomers were present in the canopy. Nitrogen concentration had the opposite trend because older phytomers comprised a greater percentage of the total culm biomass, and new tissue was produced at a greater $\mathrm{N}$ concentration before declining steadily as tissue aged. Initial growth of tissues (blades, sheaths, or internodes) typically had $\mathrm{N}$ concentrations $>50 \mathrm{~g} \mathrm{~N} \mathrm{~kg}^{-1}$ and declined as tissue aged. Identical phytomer positions tended to have similar $\mathrm{N}$ concentrations across culms: primary tillers had greater dry weights than the MC and the secondary Tiller T11 for a particular phytomer position. Mature phytomers increased in $\mathrm{N}$ mass and dry weight acropetally on a culm. This study shows that we can increase our understanding of canopy $\mathrm{N}$ dynamics and dry weight distribution patterns when we view the canopy as the interplay of the appearance, growth, interaction, and senescence of either culms or phytomers.

\section{REFERENCES}

Badaruddin, M., M.P. Reynolds, and O.A.A. Ageeb. 1999. Wheat management in warm environments: Effect of organic and inorganic fertilizers, irrigation frequency, and mulching. Agron. J. 91: 975-983.

Baker, J.M., and B.B. Tucker. 1973. Critical N, P and K levels in winter wheat. Commun. Soil Sci. Plant Anal. 4:347-358. 
Bauer, A., A.B. Frank, and A.L. Black. 1987. Aerial parts of hard red spring wheat: II. Nitrogen and phosphorus concentration and content by plant development stage. Agron. J. 79:852-858.

Blum, A., and C.Y. Sullivan. 1997. The effect of plant size on wheat response to agents of drought stress: I. Root drying. Aust. J. Plant Physiol. 24:35-41.

Boatwright, G.O., and H.J. Haas. 1961. Development and composition of spring wheat as influenced by nitrogen and phosphorus fertilization. Agron. J. 53:33-36.

Campbell, C.A., and H.R. Davidson. 1979. Effect of temperature, nitrogen fertilization and moisture stress on growth, assimilate distribution and moisture use by Manitou spring wheat. Can. J. Plant Sci. 59:603-626.

Demotes-Mainard, S., M.-H. Jeuffroy, and S. Robin. 1999. Spike dry matter and nitrogen accumulation before anthesis in wheat as affected by nitrogen fertilizer: Relationship to kernels per spike. Field Crops Res. 64:249-259.

Ehdaie, B. 1995. Variation in water-use efficiency and its components in wheat: II. Pot and field experiments. Crop Sci. 35:1617-1626.

Gallagher, J.N. 1979. Field studies of cereal leaf growth: I. Initiation and expansion in relation to temperature and ontogeny. J. Exp. Biol. 30:625-636.

Gregory, P.J., D.V. Crawford, and M. McGowan. 1979. Nutrient relations of winter wheat: I. Accumulation and distribution of $\mathrm{Na}, \mathrm{K}$, Ca, Mg, P, S, and N. J. Agric. Sci. Camb. 93:485-494.

Gregory, P.J., B. Marshall, and P.V. Biscoe. 1981. Nutrient relations of winter wheat: III. Nitrogen uptake, photosynthesis of flag leaves and translocation of nitrogen to grain. J. Agric. Sci. Camb. 96: 539-547.

Harper, J.L. 1977. Population biology of plants. Academic Press, London.

Haun, J.R. 1973. Visual quantification of wheat development. Agron. J. 65:116-119.

Hay, R.K.M., and G.T. Wilson. 1982. Leaf appearance and extension in field-grown winter wheat plants: The importance of soil temperature during vegetative growth. J. Agric. Sci. Camb. 99:403-410.

Jeuffroy, M.-H., and C. Bouchard. 1999. Intensity and duration of nitrogen deficiency on wheat grain number. Crop Sci. 39:1385-1393.

Jones, C.A., and J.R. Kiniry (ed.). 1986. CERES-Maize. A simulation model of maize growth and development. Texas A\&M Univ. Press, College Station, TX.

Karlen, D.L., and D.A. Whitney. 1980. Dry matter accumulation, mineral concentrations, and nutrient distribution in winter wheat Agron. J. 72:281-288.

Kirby, E.J.M. 1988. Analysis of leaf, stem and ear growth in wheat from terminal spikelet stage to anthesis. Field Crops Res. 18:127-140.

Klepper, B., R.W. Rickman, and R.K. Belford. 1983. Leaf and tiller identification on wheat plants. Crop Sci. 23:1002-1004.

Klepper, B., R.W. Rickman, and C.M. Peterson. 1982. Quantitative characterization of vegetative development in small cereal grains. Agron. J. 74:789-792.

Lauer, J.G., and S.R. Simmons. 1985. Photoassimilate partitioning of main shoot leaves in field-grown spring barley. Crop Sci. 25: $851-855$

Lauer, J.G., and S.R. Simmons. 1988. Photoassimilate partitioning by tillers and individual tiller leaves in field-grown spring barley. Crop Sci. 28:279-282.
McMaster, G.S. 1997. Phenology, development, and growth of the wheat (Triticum aestivum L.) shoot apex: A review. Adv. Agron. 59:63-118.

McMaster, G.S., J.A. Morgan, and W.W. Wilhelm. 1992. Simulating winter wheat spike development and growth. Agric. For. Meteorol. 60:193-220.

McMaster, G.S., W.W. Wilhelm, and P.N.S. Bartling. 1994. Irrigation and culm contribution to yield and yield components of winter wheat. Agron. J. 86:1123-1127.

McNeal, F.H., M.A. Berg, and C.A. Watson. 1966. Nitrogen and dry matter in five spring wheat varieties at successive stages of development. Agron. J. 58:605-608.

Melsted, S.W., H.L. Motto, and T.R. Peck. 1969. Critical plant nutrient composition values useful in interpreting plant analysis data Agron. J. 61:17-20.

Miller, E.C. 1939. A physiological study of the winter wheat plant at different stages of its development. Kansas Agric. Exp. Stn. Tech. Bull. 47.

Power, J.F., and J. Alessi. 1978. Tiller development and yield of standard and semidwarf spring wheat varieties as affected by nitrogen fertilizer. J. Agric. Sci. Camb. 90:97-108.

Rawson, H.M., and G. Hofstra. 1969. Translocation and remobilization of ${ }^{14} \mathrm{C}$ assimilated at different stages by each leaf of the wheat plant. Aust. J. Biol. Sci. 22:321-331.

Rickman, R.W., S.E. Waldman, and B. Klepper. 1996. MODWht3: A development-driven wheat growth simulation. Agron. J. 88: 176-185.

SAS Institute. 1989. SAS/STAT user's guide. Version 6. 4th ed. Vol. 2. SAS Inst., Cary, NC.

Schepers, J.S., D.D. Francis, and M.T. Thompson. 1989. Simultaneous determination of total $\mathrm{C}$, total $\mathrm{N}$, and $15 \mathrm{~N}$ on soil and plant material. Commun. Soil Sci. Plant Anal. 20:949-959.

Skinner, R.H., J.D. Hanson, and J.G. Benjamin. 1999. Nitrogen uptake and partitioning under alternate-and every-row irrigation. Plant Soil 210:11-20.

Spratt, E.D., and J.K.R. Gasser. 1970. Effects of fertilizer-nitrogen and water supply on distribution of dry matter and nitrogen between the different parts of wheat. Can. J. Plant Sci. 50:613-625.

Thorne, G.N. 1982. Distribution between parts of the main shoot and the tillers of photosynthate produced before and after anthesis in the top three leaves of main shoots of Hobbit and Maris Huntsman winter wheat. Ann. Appl. Biol. 101:553-559.

Waldren, R.P., and A.D. Flowerday. 1979. Growth stages and distribution of dry matter, N, P, and $\mathrm{K}$ in winter wheat. Agron. J. 71: 391-397.

Wardlaw, I.F. 1967. The effect of water stress on translocation in relation to photosynthesis and growth. Aust. J. Biol. Sci. 20:25-39.

Wilhelm, W.W., and G.S. McMaster. 1995. Importance of the phyllochron in studying development and growth in grasses. Crop Sci. 35:1-3.

Wilhelm, W.W., and G.S. McMaster. 1996. Spikelet and floret naming scheme for grasses with a spike inflorescence. Crop Sci. 36: 1071-1073.

Wilhelm, W.W., G.S. McMaster, R.W. Rickman, and B. Klepper. 1993. Above-ground vegetative development and growth of winter wheat as influenced by nitrogen and water availability. Ecol. Modell. 68:183-203. 